\title{
Efficient quantum-state tomography for quantum-information processing using a two-dimensional Fourier-transform technique
}

\author{
Ranabir Das, ${ }^{1}$ T. S. Mahesh, ${ }^{1}$ and Anil Kumar ${ }^{1,2, *}$ \\ ${ }^{1}$ Department of Physics, Indian Institute of Science, Bangalore 560012, India \\ ${ }^{2}$ Sophisticated Instruments Facility, Indian Institute of Science, Bangalore 560012, India
}

\begin{abstract}
A method of quantum-state tomography for quantum-information processing is described. The method is based on the use of the Fourier-transform technique and involves detection of all the diagonal elements of the density matrix in a one-dimensional experiment and all the off-diagonal elements by a two-dimensional experiment. The method is efficient for a large number of qubits $(\geqslant 5)$. The proposed method is outlined using a two-qubit system and demonstrated using simulations by tomographing arbitrary complex density matrices of two- and four-qubit systems.
\end{abstract}

\section{INTRODUCTION}

Quantum computation offers exciting possibilities for solving complex computational problems using algorithms which exploit the quantum nature of the system. The idea, first proposed by Feynman [1], is being feverishly pursued by many [2-6]. Several algorithms, such as Shor's factorization algorithm, Grover's search algorithm, the Deutsch-Jozsa algorithm, quantum Fourier transform, quantum counting and quantum error-correction codes have been developed and have clearly established the premise [7-24]. The last step in quantum-information processing and quantum simulations is the measurement of the output quantum state. In the case of ensemble systems this amounts to measuring the output density matrix. The output state of a quantum algorithm normally corresponds to some classical information and therefore, it is sufficient to measure all the diagonal elements of the density matrix which corresponds to the probabilities of various eigenstates. However, full quantum-state tomography is generally carried out wherever possible because of the following reasons: (i) knowledge of the full output density matrix allows one to find out the experimental errors and to calculate the fidelity of the implementation $[5,10-14]$ and (ii) if one wishes to monitor the flow of the implementation of an algorithm at any intermediate step, then the best option is to measure the full intermediate density matrix [11].

For an $n$-qubit ensemble system, the size of the Hilbert space increases as $2^{n}$ and the number of density-matrix elements increases as $2^{n} \times 2^{n}$. Of these there are $M$ $=\left(2^{n}-1\right)\left(2^{n-1}+1\right)$ independent elements, of which $\left(n 2^{n-1}\right)$ elements are single-qubit single-quantum observable elements. To measure the remaining elements, a series of one-dimensional experiments with readout pulses to rotate the unobservables into observables have been proposed earlier $[10,11,13,14]$. Here, we propose a method for quantumstate tomography based on the two-dimensional Fourier-

\footnotetext{
*Author to whom correspondence should be addressed. Email address: anilnmr@physics.iisc.ernet.in
}

transform technique, where all the off-diagonal elements of a density matrix, both observable and unobservable, are measured in a two-dimensional experiment. All the diagonal elements are measured in another one-dimensional experiment.

It has been pointed out that the earlier method of tomography involving a large number of different measurements works well for a small number of qubits, while it becomes prohibitively complex for a large number of qubits $[10,25]$. Such large systems can be easily tomographed using the method proposed here. However, the proposed method uses a two-dimensional experiment and requires a minimum number of $t_{1}$ increments (of the order of 512). In principle, the size of a two-dimensional dataset is independent of the number of qubits and all the off-diagonal elements can be detected by a fixed data size and experimental time. However, as the number of qubits increases, the number of highest quantum increases linearly. This causes a corresponding increase in spectral width in $t_{1}$ domain, requiring a corresponding increase in number of $t_{1}$ increments to maintain the same digital resolution. The maximum $t_{1}$ value is, however, decided by the total coherence time. While the number of transitions rapidly increase with the number of qubits, the coherence time is a function of inherent linewidths. Thus, the total experimental time of the proposed experimental scheme is more than the earlier method for small number of qubits but becomes less for larger number of qubits $(\geqslant 5)$. The earlier method needs independent experiments of the order of $M$, which increases exponentially with number of qubits (for four qubits $M=135$ and for five qubits $M=527$ ). The proposed method can also be used for tomography in a wider range of spin systems, such as quadrupolar or strongly coupled systems [19-23]. Furthermore, the present method requires nonselective rf pulses that are devoid of errors caused by the selective pulses used in the earlier method [10]. The method is explained here using a two-qubit system and demonstrated on the two- and four-qubit systems using simulations.

It may be recalled that the above mentioned advantage of the two-dimensional Fourier transform technique has been successfully utilized in structure determination of biomolecules by NMR [26-30]. 


\section{THE METHOD}

The method is based on the technique of indirect detection of multiple quantum coherences in NMR spectroscopy by the two-dimensional Fourier-transform technique [27], wherein all the off-diagonal elements are measured in a twodimensional experiment [pulse sequence $1(A)$ ]. The diagonal elements of the density matrix are measured in another one-dimensional experiment [pulse sequence $1(B)]$. The NMR pulse sequences for the two experiments are

$$
\begin{gathered}
(A) \sigma(0)-t_{1}-(\pi / 2)_{y}-G_{z}-\alpha_{-y}-t_{2}, \\
(B) \sigma(0)-G_{z}-\beta_{y}-t,
\end{gathered}
$$

where $t_{1}$ and $t_{2}$ are the variable time periods of system evolution, $(\pi / 2)_{y}, \alpha_{-y}$ and $\beta_{y}$ are the $\mathrm{rf}$ pulses, and $G_{z}$ is the field-gradient pulse.

In experiment $1(A)$, a given density matrix $\sigma(0)$ is allowed to evolve for a time $t_{1}$, at the end of which a $(\pi / 2)_{y}$ pulse transform every element into all other elements of the density matrix, including diagonal elements. The $G_{z}$ pulse dephases the off-diagonal elements, averaging them to zero, and retains only the diagonal elements. A $\alpha_{-y}$ pulse transforms the diagonal elements into all elements of the density matrix including single-qubit single-quantum coherences. These single-quantum coherences are then detected as a function of time variable $t_{2}$. A series of experiments are performed by systematic increment of the $t_{1}$ period and the collected two-dimensional time-domain dataset $s\left(t_{1}, t_{2}\right)$ is double Fourier transformed yielding a two-dimensional frequency domain spectrum $S\left(\Omega_{1}, \Omega_{2}\right) . S\left(\Omega_{1}, \Omega_{2}\right)$ contains along $\Omega_{2}$ all single-qubit single-quantum coherences and along $\Omega_{1}$, contribution of every off-diagonal element of the density matrix to these transitions, dispersed and displayed by their specific frequency of evolution in the time domain $t_{1}$. Cross sections parallel to $\Omega_{1}$ at each single-quantum resonance frequency can be fitted to $\sigma(0)$, yielding all the off-diagonal elements in single two-dimensional experiment. The diagonal elements of $\sigma(0)$ do not contribute to this spectrum.

Experiment 1 $(B)$ measures the diagonal elements. The given experiment begins by destroying all the off-diagonal elements of $\sigma(0)$ by a gradient pulse, and uses a small-angle pulse to convert, within linear response, difference in diagonal elements into observable single-qubit single-quantum coherences which are detected during the period $t$ and Fourier analyzed. The amplitudes of the coherences allow calculation of all the diagonal elements. The above protocol is explained in the following by explicit calculations on a two-qubit system.

\section{TWO-QUBIT SYSTEM}

Consider a two-qubit system consisting of two spin- $\frac{1}{2}$ nuclei of Larmor frequencies $\omega_{1}$ and $\omega_{2}$, coupled by a weak indirect coupling $J$. The Hamiltonian for the system is

$$
\mathcal{H}=\omega_{1} I_{1 z}+\omega_{2} I_{2 z}+J I_{1 z} I_{2 z}
$$

where $I_{j z}(j=1,2)$ are the spin operators. A selective rf pulse of angle $\theta$ and phase $\phi$ on-resonance on spin $j$ corresponds to a unitary transform

$$
U_{\theta, \phi}^{j}=\exp \left\{-i \theta\left(I_{j x} \sin \phi+I_{j y} \cos \phi\right)\right\} .
$$

All quantum algorithms are implemented in NMR by a specific pulse sequence involving the evolutions under system Hamiltonian and rf pulses. A general Hermitian complex traceless deviation density matrix for two qubits has 15 independent elements, is spanned by 15 product operators [26], and can be expressed as

$$
\sigma(0)=\sum_{k, l} q_{k l} I_{1 k} I_{2 l}
$$

where $k$ and $l$ can take values $0,1,2,3$ corresponding to $o, x$, $y, z$, respectively, but not simultaneously $0 . I_{1 o}=I_{2 o}=I$ is a unit matrix and $q_{k l}$ are real coefficients. All the elements of the density matrix $\sigma(0)$ can be classified into two groups; (i) diagonal elements involving deviation populations $\Delta P_{k}$ (deviations from an average population) of various eigenstates and (ii) off-diagonal elements involving one-qubit coherences (single quantum elements) and multiqubit coherences (zero- and double-quantum elements for a two qubit system). The density matrix can be depicted as

$$
\sigma(0)=\left(\begin{array}{cccc}
\Delta P_{1} & S_{1} & S_{2} & D \\
S_{1}^{*} & \Delta P_{2} & Z & S_{3} \\
S_{2}^{*} & Z^{*} & \Delta P_{3} & S_{4} \\
D^{*} & S_{3}^{*} & S_{4}^{*} & \Delta P_{4}
\end{array}\right) .
$$

The trace condition $\Sigma_{k} \Delta P_{k}=0$ yields 15 independent (12 off-diagonal and three diagonal) elements in this case. Each single-quantum element corresponds to linear combinations of operators of the type $I_{1 x}, I_{1 y}, I_{1 x} I_{2 z}, I_{1 y} I_{2 z}$, and $I_{2 x}$, $I_{2 y}, I_{2 x} I_{1 z}$, and $I_{2 y} I_{1 z}$. Each two-quantum element, known as zero- or double-quantum element $(Z, D)$, corresponds to linear combinations of product operators $I_{1 x} I_{2 x}, I_{1 x} I_{2 y}$, $I_{1 y} I_{2 x}$, and $I_{1 y} I_{2 y}$.

\section{A. Measurement of the off-diagonal elements}

The evolution of $\sigma(0)$ during the pulse sequence 1(A) can be described as [26,27],

$$
\begin{aligned}
& \sigma(0) \stackrel{t_{1}}{\rightarrow} \sigma_{1}\left(t_{1}\right)=e^{-i \mathcal{H} t_{1}} \sigma(0) e^{i \mathcal{H} t_{1}} \\
& (\pi / 2)_{y} \\
& \quad \rightarrow \sigma_{2}\left(t_{1}\right)=e^{-i\left(I_{1 y}+I_{2 y}\right) \pi / 2} \sigma_{1}\left(t_{1}\right) e^{i\left(I_{1 y}+I_{2 y}\right) \pi / 2} \\
& \quad G_{z} \\
& \quad \rightarrow \sigma_{3}\left(t_{1}\right)=\mathcal{P} \sigma_{2}\left(t_{1}\right) \\
& \quad \alpha_{-y} \\
& \quad \rightarrow \sigma_{4}\left(t_{1}\right)=e^{i\left(I_{1 y}+I_{2 y}\right) \alpha} \sigma_{3}\left(t_{1}\right) e^{-i\left(I_{1 y}+I_{2 y}\right) \alpha} \\
& t_{2}(\text { measure }) \\
& \rightarrow \quad \sigma_{5}\left(t_{1}, t_{2}\right)=e^{-i \mathcal{H} t_{2}} \sigma_{4}\left(t_{1}\right) e^{i \mathcal{H} t_{2} .} .
\end{aligned}
$$

Here, the operator $\mathcal{P}$ projects and retains only the diagonal part of $\sigma_{2}\left(t_{1}\right)$. The complex time domain signal $s\left(t_{1}, t_{2}\right)$ 
obtained on measurement as a function of $t_{2}$, is $s\left(t_{1}, t_{2}\right)$ $=\operatorname{Tr}\left[\left(I_{1}^{+}+I_{2}^{+}\right) \sigma_{5}\left(t_{1}, t_{2}\right)\right]$, which after double Fourier transform gives two-dimensional (2D) spectrum $S\left(\Omega_{1}, \Omega_{2}\right)$ which is a function of the two frequency variables $\Omega_{1}$ and $\Omega_{2}[26,27]$. The $\Omega_{2}$ axis of this spectrum has only the singlequantum (1Q) elements (four transitions in the two-qubit system). Along the $\Omega_{1}$ axis of the $2 \mathrm{D}$ spectrum all the offdiagonal elements of $\sigma(0)$ yield peaks corresponding to their amplitudes $q_{k l}$ and individual evolution frequencies during the time period $t_{1}$.

Cross sections of the signal after Fourier transform with respect to $t_{2}$, but before $t_{1},\left[S\left(t_{1}, \Omega_{1}\right)\right]$ taken parallel to $t_{1}$ at $\Omega_{2}=\omega_{1}^{+}$and $\Omega_{2}=\omega_{2}^{+}$, respectively, correspond to

$$
\begin{aligned}
\sigma_{4}\left(\omega_{1}^{+}\right)= & e^{-t_{1} / T_{2}} \sin \alpha\left[\left(q_{10}+q_{13}\right) \cos \left(\omega_{1}^{+} t_{1}\right)\right. \\
& +\left(q_{10}-q_{13}\right) \cos \left(\omega_{1}^{-} t_{1}\right)-\left(q_{20}+q_{23}\right) \sin \left(\omega_{1}^{+} t_{1}\right) \\
& -\left(q_{20}-q_{23}\right) \sin \left(\omega_{1}^{-} t_{1}\right) \\
& +\frac{1}{2} \cos \alpha\left\{\left(q_{11}-q_{22}\right) \cos \left(\omega^{D} t_{1}\right)\right. \\
& +\left(q_{11}+q_{22}\right) \cos \left(\omega^{Z} t_{1}\right)+\left(q_{12}+q_{21}\right) \sin \left(\omega^{D} t_{1}\right) \\
& \left.\left.-\left(q_{12}-q_{21}\right) \sin \left(\omega^{Z} t_{1}\right)\right\}\right] / 4,
\end{aligned}
$$

$$
\begin{aligned}
\sigma_{4}\left(\omega_{2}^{+}\right)= & e^{-t_{1} / T_{2}} \sin \alpha\left[\left(q_{01}+q_{31}\right) \cos \left(\omega_{2}^{+} t_{1}\right)\right. \\
& +\left(q_{01}-q_{31}\right) \cos \left(\omega_{2}^{-} t_{1}\right)-\left(q_{02}+q_{32}\right) \sin \left(\omega_{2}^{+} t_{1}\right) \\
& -\left(q_{02}-q_{32}\right) \sin \left(\omega_{2}^{-} t_{1}\right) \\
& +\frac{1}{2} \cos \alpha\left\{\left(q_{11}-q_{22}\right) \cos \left(\omega^{D} t_{1}\right)\right. \\
& +\left(q_{11}+q_{22}\right) \cos \left(\omega^{Z} t_{1}\right)+\left(q_{12}+q_{21}\right) \sin \left(\omega^{D} t_{1}\right) \\
& \left.\left.-\left(q_{12}-q_{21}\right) \sin \left(\omega^{Z} t_{1}\right)\right\}\right] / 4,
\end{aligned}
$$

where $\omega_{j}^{ \pm}=\omega_{j} \pm J / 2, \omega^{D}=\omega_{1}+\omega_{2}, \omega^{Z}=\omega_{1}-\omega_{2}, q_{k l}$ are the coefficients of expansion of $\sigma(0)$ as in Eq. (4), and $T_{2}$ is the transverse relaxation time of various coherences. $T_{2}$ can be different for each coherence but is for simplicity, taken to be identical here. On $t_{1}$ Fourier transformation of expression (7), one obtains the two-dimensional spectrum $S\left(\Omega_{1}, \Omega_{2}\right)$ in which the cosine terms give absorptive and the sine terms dispersive Lorenztian lines. All the coefficients of the offdiagonal elements of $\sigma(0)$ can be obtained by fitting the cross sections from the two-dimensional spectrum $S\left(\Omega_{1}, \Omega_{2}\right)$ (taken parallel to $\left.\Omega_{1}\right)$ to the absorptivedispersive Lorentzians obtained from expressions similar to Eq. (7). Two cross sections, one at each qubit, are sufficient to calculate all the off-diagonal elements of the two-qubit density matrix $\sigma(0)$ of Eq. (4). For an $n$-qubit system, there are $2^{n-1}$ cross sections per qubit. While only one crosssection per qubit is required to map all the off-diagonal elements, the remaining cross sections can be used to minimize the errors. It may also be noted that the diagonal elements do not interfere with the two-dimensional spectrum obtained by pulse sequence $1(A)$. Similarly the undesired "axial peaks" (having zero frequency during $t_{1}$ period) arising due to the longitudinal relaxations during $t_{1}$ period [26] are also suppressed by the present scheme.

In the two-qubit case, the one-qubit and two-qubit coherences have different conversion ratios in the detected signal, respectively, $\sin \alpha$ and $\frac{1}{4} \sin 2 \alpha$. To optimize both these signals $\alpha=\pi / 4$ has been used here. In the back calculation of $\sigma(0)$, these factors have to be taken into account. For higher qubit systems having higher quantum coherences, appropriate values of $\alpha$ should be used that optimize the intensities of various orders [31].

\section{B. Measurement of the diagonal elements}

The evolution of $\sigma(0)$ during the pulse sequence $1(B)$ can be written as

$$
\begin{gathered}
\stackrel{G_{z}}{\sigma(0)} \underset{\beta_{y}}{\rightarrow} \sigma_{1}=\mathcal{P} \sigma(0) \\
\stackrel{\rightarrow}{\rightarrow} \sigma_{2}=e^{-i\left(I_{1 y}+I_{2 y}\right) \beta} \sigma_{1} e^{i\left(I_{1 y}+I_{2 y}\right) \beta} \\
\stackrel{\text { (measure })}{\rightarrow} \sigma_{3}=e^{-i \mathcal{H} t} \sigma_{2} e^{i \mathcal{H} t},
\end{gathered}
$$

where

$$
\sigma_{1}=q_{30} I_{1 z}+q_{03} I_{2 z}+q_{33} I_{1 z} I_{2 z},
$$

and

$$
\begin{aligned}
\sigma_{2}= & q_{30}\left(I_{1 z} \cos \beta+I_{1 x} \sin \beta\right)+q_{03}\left(I_{2 z} \cos \beta+I_{2 x} \sin \beta\right) \\
& +q_{33}\left(I_{1 z} \cos \beta+I_{1 x} \sin \beta\right)\left(I_{2 z} \cos \beta+I_{2 x} \sin \beta\right) .
\end{aligned}
$$

To measure the coefficients under linear response, $\beta$ should be small [26]. The measured one-qubit coherences then are

$$
\sigma_{2}(\beta \rightarrow 0)=\beta\left[q_{30} I_{1 x}+q_{03} I_{2 x}+q_{33}\left(I_{1 x} I_{2 z}+I_{1 z} I_{2 x}\right)\right],
$$

which can be rearranged as

$$
\begin{aligned}
\sigma_{2}(\beta \rightarrow 0)= & \beta\left[\left(q_{30}+q_{33} / 2\right)\left(I_{1 x}+2 I_{1 x} I_{2 z}\right)\right. \\
& +\left(q_{30}-q_{33} / 2\right)\left(I_{1 x}-2 I_{1 x} I_{2 z}\right) \\
& +\left(q_{03}+q_{33} / 2\right)\left(I_{2 x}+2 I_{1 z} I_{2 x}\right) \\
& \left.+\left(q_{03}-q_{33} / 2\right)\left(I_{2 x}-2 I_{1 z} I_{2 x}\right)\right] / 2 .
\end{aligned}
$$


(a)

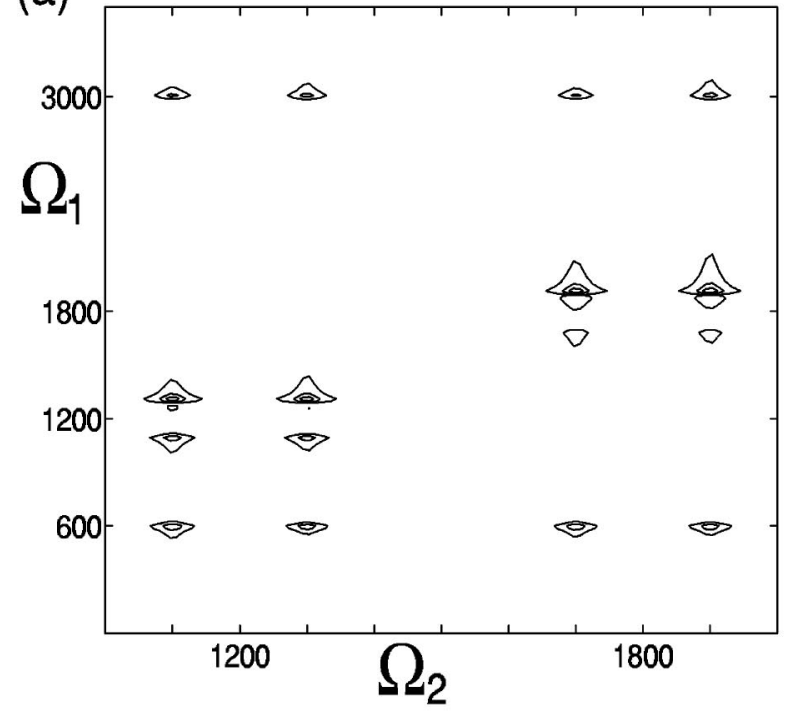

(e)

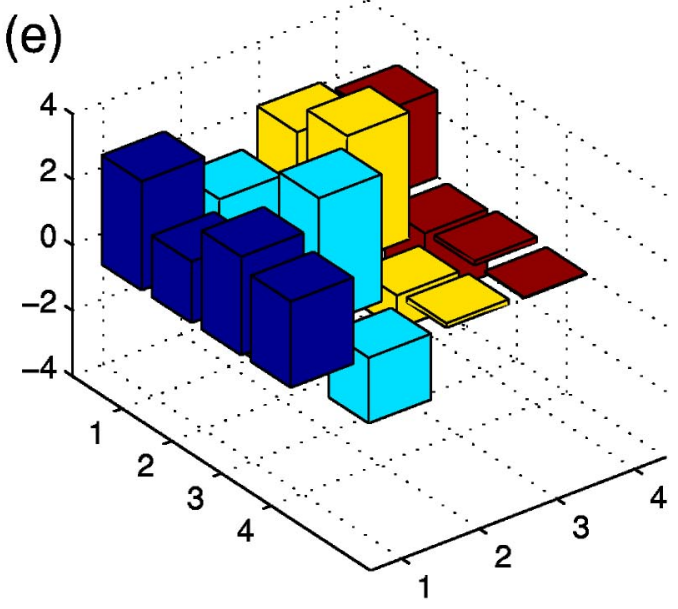

(b)

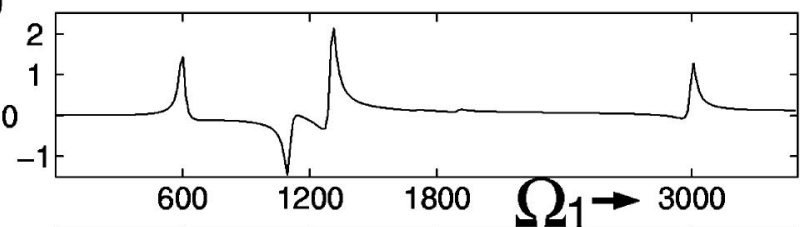

(c)

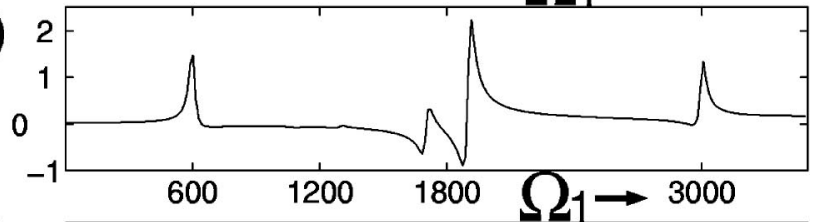

(d)

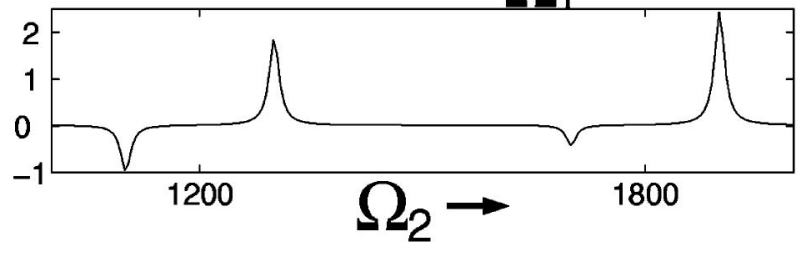

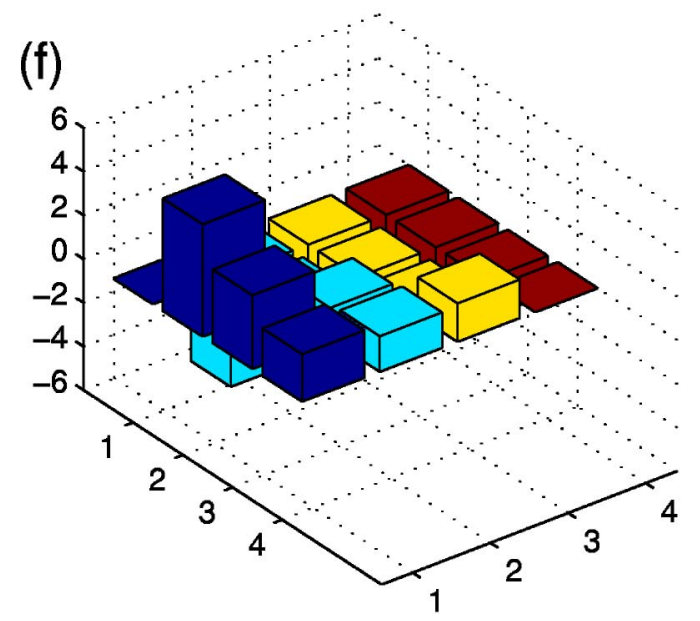

FIG. 1. Tomography of a complex density matrix in a two-qubit system. (a) is the $2 \mathrm{D}$ spectrum generated by experiment $1(A)$ for tomography of all the off-diagonal elements, (b) is the cross section parallel to $\Omega_{1}$ taken at $\Omega_{2}=1300 \mathrm{~Hz}\left(\omega_{1}+J / 2\right)$ (a transition frequency of first qubit), (c) is the cross section parallel to $\Omega_{1}$ taken at $\Omega_{2}=1900 \mathrm{~Hz}\left(\omega_{2}+J / 2\right)$ (a transition frequency of the second qubit), and (d) is the $1 \mathrm{D}$ spectrum obtained by experiment of $1(B)$ for mapping all diagonal elements of $\sigma(0) . \Omega_{1}$ and $\Omega_{2}$ are in units of hertz for all the spectra. (e) and (f) are the real and imaginary parts of the tomographed density matrix. The $x$ and $y$ axes are indices of the elements of the density matrix and their respective intensities are along the $z$ axis. The calculated values of the tomographed matrix are $\sigma_{11}=3.32501$, $\sigma_{12}=1.8625-5.1385 i, \sigma_{13}=3.0001-3.375 i, \sigma_{14}=2.6501-2.1624 i, \sigma_{21}=1.8625+5.1385 i, \sigma_{22}=-2.3251, \sigma_{23}=3.8750+1.4374 i, \sigma_{24}$ $=-2.0001-1.625 i, \quad \sigma_{31}=3.0001+3.375 i, \quad \sigma_{32}=3.8750-1.4374 i, \quad \sigma_{33}=-1.025, \quad \sigma_{34}=0.1376-1.7618 i, \quad \sigma_{41}=2.6501+2.1624 i, \quad \sigma_{42}$ $=-2.0001+1.625 i, \sigma_{43}=0.1376+1.7618 i$, and $\sigma_{44}=0.025$.

The coefficients of the four terms in the above expression are proportional to the intensities of the corresponding four transitions of a two-qubit system. After calculating $q_{30}, q_{03}$, and $q_{33}$, all the diagonal elements of the density matrix $\sigma(0)$ can be calculated, since the diagonal part is equal to $q_{30} I_{1 z}$ $+q_{03} I_{2 z}+q_{33} I_{1 z} I_{2 z}$.

It should be noted that the gradient pulse $G_{z}$ used in pulse sequence $1(A)$ to destroy the off-diagonal elements, does not destroy homonuclear zero-quantum coherences. In such cases, an extra small delay $\tau_{m}$ along with $G_{z}$, randomly varied between each $t_{1}$ experiment can suppress the homo- nuclear zero-quantum coherence [26]. In experiment $1(B)$, signal averaging using a few randomly varied $\tau_{m}$ along with $G_{z}$ would suppress the homonuclear zero-quantum coherences [26].

The above schemes $1(A)$ and $1(B)$ assume ideal $\mathrm{rf}$ pulses. To correct for errors due to imperfection of rf pulses, a direct measurement (one dimensional) of $\sigma(0)$ yields the amplitudes of all single-quantum single-qubit coherences. These amplitudes can then be used to normalize all elements of the density matrix measured by experiments $1(A)$ and $1(B)$. 
INPUT

REAL

(a)

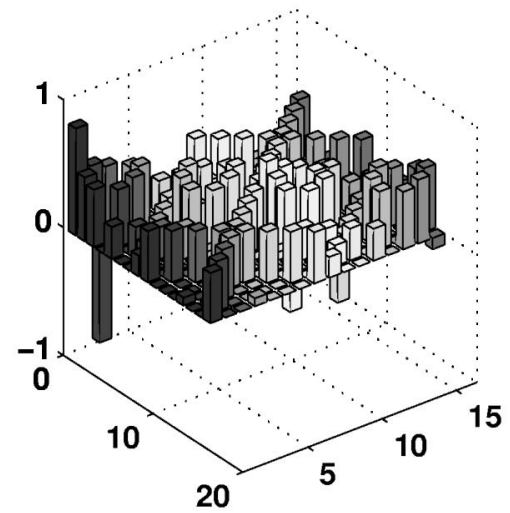

20

IMAG

(b)

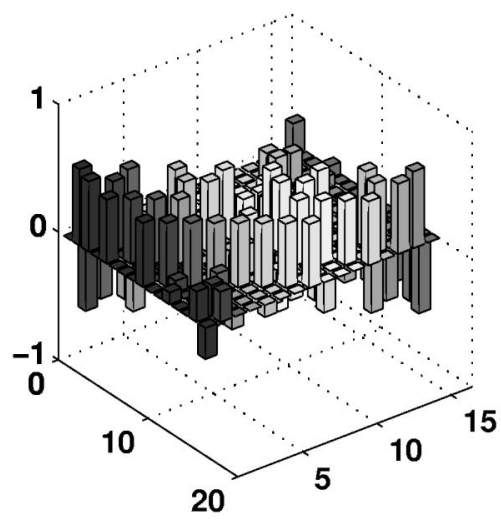

RECONSTRUCTED

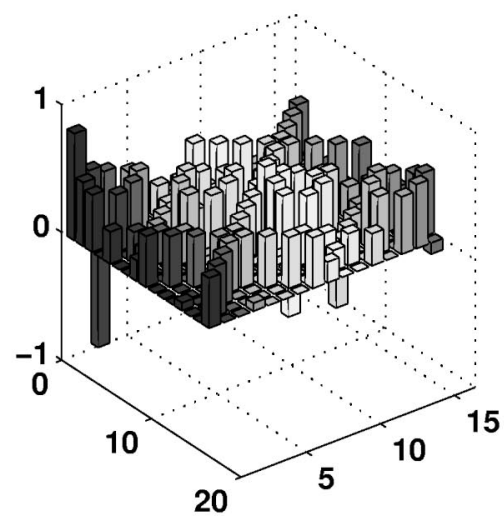

(c)

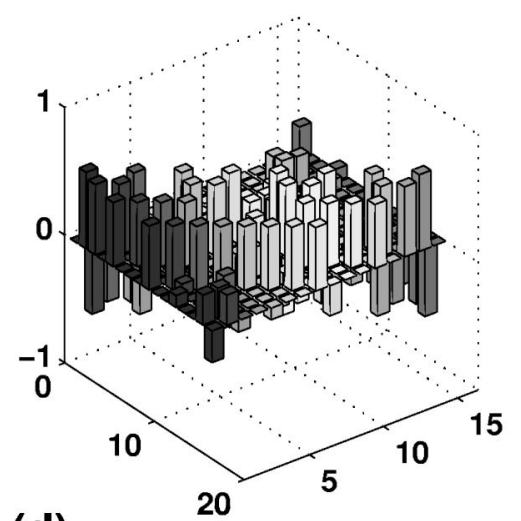

(d)
DIFFERENCE $\left(10^{3}\right)$

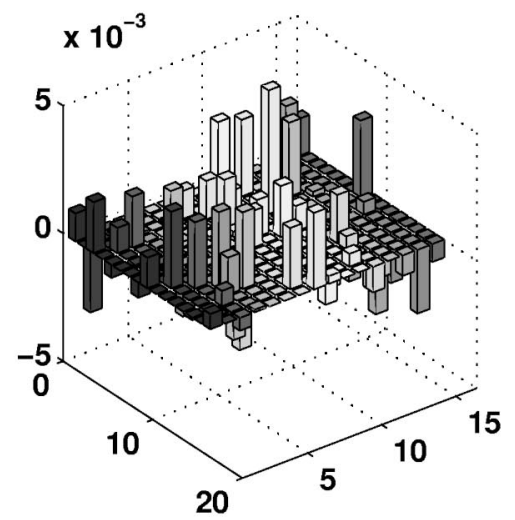

(e)

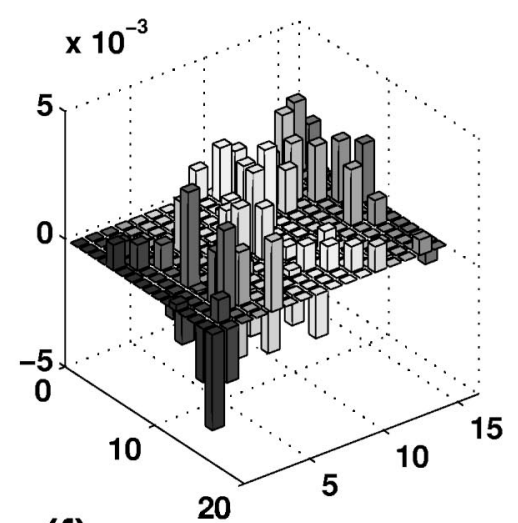

(f)

FIG. 2. Tomography of a complex density matrix in a four-qubit system. An arbitrary complex density matrix $\sigma(0)=0.8 I_{1 x}+I_{1 y}$ $+0.5 I_{2 x}+I_{2 y}+0.9 I_{3 x}+1.1 I_{3 y}+I_{4 x}+1.2 I_{4 y}+6.3 I_{1 x} I_{2 x} I_{3 x} I_{4 x}+3.9 I_{1 x} I_{2 y} I_{3 y} I_{4 y}+I_{1 x} I_{2 x} I_{3 z} I_{4 z}+1.3 I_{2 x} I_{3 x} I_{4 x}+1.9 I_{1 x} I_{2 x} I_{3 y}+1.5 I_{1 x} I_{2 z} I_{3 y} I_{4 x}$ $+0.6 I_{4 z}+I_{2 z} I_{4 z}+1.3 I_{2 z} I_{3 z} I_{4 z}+2 I_{1 z} I_{2 z} I_{3 z} I_{4 z}$ is reconstructed with the $2 \mathrm{D}$ Fourier-transform technique. The frequencies and couplings used in the simulation are $\omega_{1}=600 \mathrm{~Hz}, \omega_{2}=750 \mathrm{~Hz}, \omega_{3}=1000 \mathrm{~Hz}, \omega_{4}=1400 \mathrm{~Hz}, J_{12}=20 \mathrm{~Hz}, J_{13}=10 \mathrm{~Hz}, J_{14}=70 \mathrm{~Hz}, J_{23}=35 \mathrm{~Hz}, J_{24}$ $=24 \mathrm{~Hz}$, and $J_{34}=16 \mathrm{~Hz}$. (a) Real and (b) imaginary parts of $\sigma(0)$. (c) and (d) are the real and imaginary parts of the reconstructed density matrix. (e) is the difference (magnified by $10^{3}$ ) between the matrices of (a) and (c), while (f) is the difference between (b) and (d). The $x$ and $y$ axes are indices of the elements of the density matrix and their respective intensities are along the $z$ axis. The 256 complex elements of $\sigma(0)$ were tomographed with $99.7 \%$ accuracy.

\section{SIMULATION}

To demonstrate the protocol, we tomograph an arbitrary complex density matrix with simulations. In a two-qubit system, an instance of general density matrix is of the form

$$
\begin{aligned}
\sigma(0)= & I_{1 z}+2.3 I_{2 z}+6.7 I_{1 z} I_{2 z}+I_{1 x}+10 I_{1 x} I_{2 z}+5 I_{1 y}+3.5 I_{1 y} I_{2 z}+2.5 I_{1 y} I_{2 y} \\
& +7.2 I_{1 y} I_{1 x}+13 I_{1 x} I_{2 x}+1.45 I_{1 x} I_{2 y}+2 I_{2 x}+3.45 I_{1 z} I_{2 x}+6.9 I_{2 y}+6.753 I_{1 z} I_{2 y} \\
= & \left(\begin{array}{cccc}
3.325 & 1.8625-5.1383 i & 3-3.75 i & 2.65-2.1625 i \\
1.8625+5.1383 i & -2.325 & 3.875-1.4375 i & -2-1.625 i \\
3+3.375 i & 3.875+1.4375 i & -1.025 & 0.1375-1.7618 i \\
2.65+2.1625 i & -2+1.625 i & 0.1375+1.7618 i & 0.025
\end{array}\right) .
\end{aligned}
$$


We assume the Larmor frequencies of the two qubits to be $\omega_{1}=1200 \mathrm{~Hz}, \omega_{2}=1800 \mathrm{~Hz}$ and the indirect coupling between the qubits to be $J=200 \mathrm{~Hz}$. Experiment $1(A)$ is performed to obtain all the off-diagonal elements. $\alpha$ was chosen as $45^{\circ} .512 t_{1}$ increments were performed yielding the $2 \mathrm{D}$ spectrum shown in Fig. 1(a). Cross sections parallel to $\Omega_{1}$, taken at one transition of each qubit, are shown in Figs. 1(b) and 1(c). These cross sections were fitted to get all the complex off-diagonal elements of the density matrix. $T_{2}$ was taken as $10 \mathrm{~ms}$ for all coherences. The diagonal elements were mapped using experiment $1(B)$ with $\beta=10^{\circ}$, Fig. 1(d). The real and imaginary parts of the tomographed density matrix are shown, respectively, in Figs. 1(e) and 1(f). The calculated density matrix matches the input density matrix better than $0.01 \%$ for all complex elements. We have also carried out the simulations on a four-qubit system and tomographed an arbitrary complex density matrix with $99.7 \%$ fidelity (Fig. 2).

\section{CONCLUSION}

The two-dimensional nuclear-magnetic-resonance spectroscopy provides an efficient method for the quantum-state tomography. Only a one-dimensional and a two-dimensional experiment are required for measuring all the elements of the density matrix. The earlier method requires a series of onedimensional experiments with different readout pulses. This approach becomes enormously complex for a large number of qubits [16]. However, the method proposed here is aptly suited for tomography of a large number of qubits $(\geqslant 5)$. It may also be mentioned that the proposed method is not system specific and can be set up easily for any number of qubits in any configuration. The earlier method uses spinselective $\mathrm{rf}$ pulses, which requires long-duration irradiation of particular spins. During such pulses, the unperturbed spins evolve under the Zeeman and coupling interactions, introducing errors due to measurement $[15,16]$. The method described here requires nonselective short-duration $\mathrm{rf}$ pulses that do not introduce such errors. Search of more qubits has led researchers to use strongly coupled spin- $\frac{1}{2}$ nuclei and quadrupolar nuclei $\left(\operatorname{spin}>\frac{1}{2}\right)$ oriented in liquid crystalline matrices [19-23]. For such systems the notion of spin selectivity does not apply, but the proposed method based on nonselective pulses can be used for tomography. Recently, the method has been used to tomograph the states during quantum-information processing in weakly and strongly coupled spin systems $[23,24]$. This method can also be extended to a three-dimensional experiment in which quantas of various orders are displaced in different planes of the 3D experiment, increasing the detectibility and the resolution of the quantas [32].

\section{ACKNOWLEDGMENTS}

Useful discussions with Professor K. V. Ramanathan and Mr. Neeraj Sinha are gratefully acknowledged.
[1] R.P. Feynman, Int. J. Theor. Phys. 21, 467 (1982).

[2] S. Lloyd, Science 273, 1073 (1996).

[3] N.A. Gershenfeld, I.L. Chuang, Science 275, 350 (1997).

[4] D.G. Cory, A.F. Fahmy, T.F. Havel, Proc. Natl. Acad. Sci. U.S.A. 94, 1634 (1997).

[5] M.A. Nielsen and I.L. Chuang, Quantum Computation and Quantum Information (Cambridge University Press, Cambridge, 2000).

[6] The Physics of Quantum Information, edited by D. Bouwnmeester, A. Ekert, A. Zeilinger (Springer, Berlin, 2000).

[7] D. Deutsch, Proc. R. Soc. London, Ser. A 400, 97 (1985).

[8] P.W. Shor, SIAM J. Comput. 26, 1484 (1997).

[9] L.K. Grover, Phys. Rev. Lett. 79, 325 (1997).

[10] I.L. Chuang, N. Greshenfeld, M. Kubinec, and D. Leung, Proc. R. Soc. London, Ser. A 454, 447 (1998).

[11] I.L. Chuang, N. Gershenfeld, and M. Kubinec, Phys. Rev. Lett. 80, 3408 (1998).

[12] M.A. Nielsen, E. Knill, and R. Laflamme, Nature (London) 396, 52 (1998).

[13] L.M.K. Vanderspypen, M. Steffen, M.H. Sherwood, C.S. Yannoni, R. Cleve, and I.L. Chuang, Appl. Phys. Lett. 76, 646 (2000).

[14] Y.S. Weinstein, M.A. Pravia, E.M. Fortunato, S. Lloyd, and D.G. Cory, Phys. Rev. Lett. 86, 1889 (2001).

[15] J.A. Jones and M. Mosca, J. Chem. Phys. 109, 1648 (1998).

[16] J.A. Jones, Prog. Nucl. Magn. Reson. Spectrosc. 38, 325 (2001).
[17] K. Dorai, Arvind, and A. Kumar, Phys. Rev. A 63, 034101 (2001).

[18] T.S. Mahesh, A. Kumar, Phys. Rev. A 64, 012307 (2001).

[19] A.K. Khitrin and B.M. Fung, J. Chem. Phys. 112, 6963 (2000).

[20] A. Khitrin, H. Sun, and B.M. Fung, Phys. Rev. A 63, 020301(R) (2001).

[21] N. Sinha, T.S. Mahesh, K.V. Ramanathan, and A. Kumar, J. Chem. Phys. 114, 4415 (2002).

[22] K.V.R.M. Murali, N. Sinha, T.S. Mahesh, M. Levitt, K.V. Ramanathan, and A. Kumar, Phys. Rev. A 66, 022313 (2002).

[23] T.S. Mahesh and A. Kumar, e-print quant-ph/0212123.

[24] R. Das, T. S. Mahesh, and A. Kumar, Chem. Phys. Lett. 369, 8 (2003).

[25] Reference [16], p. 21.

[26] R.R. Ernst, G. Bodenhausen, and A. Wokaun, Principles of Nuclear Magnetic Resonance in One and Two Dimensions (Clarendon Press, Oxford, 1987).

[27] W.P. Aue, E. Bertholdi, and R.R. Ernst, J. Chem. Phys. 64, 2229 (1976).

[28] K. Wüthrich, NMR of Proteins and Nucleic Acids (Wiley, New York, 1986).

[29] Ivan Amato, Science 254, 518 (1991).

[30] Adrian Cho and Dennis Normille, Science (Washington, DC, U.S.) 298, 527 (2002).

[31] N. Murali and A. Kumar, Chem. Phys. Lett. 137, 324 (1987).

[32] R.T. Syvitski, N. Burlinson, E.E. Burnell, and J. Jeener, J. Magn. Reson. 155, 251 (2002). 